\title{
Distribution Network Design for Fixed Lifetime Perishable Products: A Model and Solution Approach
}

\author{
Z. Firoozi, ${ }^{1}$ N. Ismail, ${ }^{1}$ Sh. Ariafar, ${ }^{2}$ S. H. Tang, ${ }^{1}$ M. K. A. M. Ariffin, ${ }^{1}$ and A. Memariani ${ }^{3}$ \\ ${ }^{1}$ Department of Mechanical and Manufacturing Engineering, University Putra Malaysia, 43400 Serdang, Selangor, Malaysia \\ ${ }^{2}$ Industrial Engineering Department, College of Engineering, Shahid Bahonar University, Kerman 7618891167, Iran \\ ${ }^{3}$ Department of Mathematics and Computer Science, University of Economic Sciences, Tehran 1593656311, Iran
}

Correspondence should be addressed to Z. Firoozi; zhr_firoozi@yahoo.com

Received 21 October 2012; Revised 16 February 2013; Accepted 27 February 2013

Academic Editor: Yuri Sotskov

Copyright (c) 2013 Z. Firoozi et al. This is an open access article distributed under the Creative Commons Attribution License, which permits unrestricted use, distribution, and reproduction in any medium, provided the original work is properly cited.

\begin{abstract}
Nowadays, many distribution networks deal with the distribution and storage of perishable products. However, distribution network design models are largely based on assumptions that do not consider time limitations for the storage of products within the network. This study develops a model for the design of a distribution network that considers the short lifetime of perishable products. The model simultaneously determines the network configuration and inventory control decisions of the network. Moreover, as the lifetime is strictly dependent on the storage conditions, the model develops a trade-off between enhancing storage conditions (higher inventory cost) to obtain a longer lifetime and selecting those storage conditions that lead to shorter lifetimes (less inventory cost). To solve the model, an efficient Lagrangian relaxation heuristic algorithm is developed. The model and algorithm are validated by sensitivity analysis on some key parameters. Results show that the algorithm finds optimal or near optimal solutions even for large-size cases.
\end{abstract}

\section{Introduction}

A considerable proportion of the products produced worldwide are perishable. For instance, $50 \%$ of sales in the US grocery industry are due to perishable products [1], and in the area of blood management, more than 92 million units of blood, which are perishable, are collected globally every year, according to the World Health Organization (WHO) [2]. Medicines, pharmaceutical products, and many industrial products are other varieties of perishable goods. Perishable products are only usable during their lifetime; when their lifetime is over, they must be discarded [3]. This lifetime must be considered when deciding on inventory control policies for perishable products [4-6]. High volume production and high sensitivity imply that, for perishable products, distribution network design (DND) is of great significance.

DND is one of the most comprehensive decision problems in logistics and supply chain management [7-10]. Formerly, DND models only considered strategic decisions, including facility location, capacity planning, and transportation mode selection. Studies by Amiri [11] and Melkote and Daskin [12] can be cited as examples of this group. Other important decisions, such as routing and inventory control, either were not intended in the distribution network design or were considered after determination of the strategic decisions and not contemporaneously. The components of cost associated with these decisions are estimated to contribute about $10 \%$ to $25 \%$ of the sale [13]. Additionally, these decisions are highly interdependent [14, 15]. For instance, location decisions have a significant impact on the inventory and transportation cost [16], such that decreasing the number of warehouses in a network, reduces inventory cost but increases transportation costs [14]. Thus, traditional approaches that only consider strategic decisions or that optimize decisions separately could overlook potentially large cost savings and improved customer satisfaction [7]. Evidence for this hypothesis can be found in the study by Miranda and Garrido [17]. Their work concluded that a simultaneous approach to optimizing inventory and facility location decisions could 
lead to greater cost savings in comparison with a sequential approach that optimizes location decisions first and inventory decisions later. Therefore, a more comprehensive concept emerged, that is integrated distribution network design, which simultaneously optimizes a wider range of decisions, including: facility location, transportation, inventory control, routing, ordering, and production scheduling. Examples of the latter group are studies by Berman et al. [18] and Tsao et al. [19].

Despite a large number of distribution networks that deal with the transportation and storage of perishable products $[5,6,20,21]$, many of the integrated network design models consider an infinite lifetime for commodities, which makes them unsuitable for perishable products $[4,22]$. However, the cost and quality of final products are strictly related to the efficiency of network design [23]. Therefore, new research is required to incorporate inventory models of perishable products into integrated distribution network design models. Accordingly, the aim of this paper is to formulate and solve an integrated inventory location model for perishable commodities with fixed lifetimes. The effect of lifetime and some other key parameters on the objective function are investigated in this study.

\section{Research Background}

A typical distribution network consists of one or more suppliers, a set of retailers, and a set of distribution centers. The distribution centers act as stocking points in the network that order products from suppliers to fulfill the demands of retailers. The inventory of several retailers is aggregated into one distribution center. The objective of an integrated location-inventory model is to determine the optimal number and location of the distribution centers, the assignment of retailers to distribution centers, and the optimal inventory level of the distribution centers, such that total transportation, inventory, and fixed installation costs are minimized [24].

One of the most cited integrated inventory location model is the location model with risk pooling (LMRP) developed by Daskin et al. [25]. This model incorporated inventory and safety stock decisions into the single product uncapacitated facility location problem (UFLP). The solution method was based on Lagrangian relaxation. Shen et al. [26] also solved the LMRP, but used a set partitioning approach. Both of these works assumed that the demands of retailers were deterministic and that the proportions of mean to the variance of demands for all retailers were identical. LMRP was then extended in different ways. A multiproduct version of LMRP was developed by Shen [27], solving the model using the Lagrangian relaxation. Shu et al. [28] solved a general case of LMRP in which the proportions of mean to the variance for retailers' demands were not identical. Sourirajan et al. [29] and Sourirajan et al. [30] developed the LMRP by removing the assumption of identical lead times between supplier and distribution centers (DCs). Qi and Shen [31] studied the effects of uncertainty on network design decisions. Max Shen and Qi [16] estimated the total routing cost of the network and incorporated it into the LMRP. The problem was solved using Lagrangian relaxation. Gebennini et al. [32] developed a dynamic version of the problem that simultaneously determined the network configuration decisions, inventory control decisions, and production rate of a network. Jha et al. [33] studied the effect of transportation costs of a joint inventory location model using a modified adaptive differential evolution algorithm. Melo et al. [34] addressed the problem of redesigning a distribution network, a context that is rarely considered in the literature. Shavandi and Bozorgi [35] considered the demand as a fuzzy variable and formulated the problem using the credibility theory in order to locate distribution centers as well as to determine inventory levels in DCs. Several joint location the inventory problems with stochastic retailer demand were also studied by Atamtürk et al. [36].

One of the disadvantages of LMRP is that this model does not consider capacity restrictions of distribution centers. Miranda and Garrido [17] presented an extension of LMRP by including capacity constraints of distribution centers into the objective function of the LMRP model. Ozsen et al. [14] and Miranda and Garrido [37] defined a new stochastic constraint based on inventory management policy. This constraint makes sure that the maximum inventory on hand in each DC does not exceed the DCs' storage capacity. Inclusion of this stochastic capacity constraint provided the tradeoff between the establishment of more warehouses (increase of fixed facility cost) versus more frequent ordering from the supplier (increase of the ordering cost) in distribution networks. Ozsen et al. [14] included stochastic DCs' capacity restrictions into the LMRP. They assumed that the demands of retailers follow a Poisson distribution. The newly derived model was called the capacitated location model with risk pooling (CLMRP) and was solved using the Lagrangian relaxation. Both of the above mentioned papers assumed an economic order quantity (EOQ) and $(Q, r)$ as the inventory policy for the distribution networks.

Another body of the literature related to this research falls under the perishable inventory control theory. According to Goyal and Giri [38], one group of perishable inventories are those that have a fixed lifetime or a predetermined expiry date. Important examples of this group of commodities are human blood, medical drugs, and most processed food. Literature on fixed lifetime perishable inventory is rich, for example, the studies by $[6,22,39,40]$. However, despite their valuable contributions, these papers did not incorporate perishable inventory control into integrated DND models. Similarly, among network design models, Daskin et al. [25], Shen et al. [26], and Shu et al. [28] stated that their studies were motivated by the work of a blood bank network, responsible for the production and distribution of one of the most perishable types of blood products. Nevertheless, the developed models in the mentioned studies did not consider the lifetime of this product.

In this paper, the Lagrangian relaxation is selected as the solution method and thus, it is worth presenting a brief review of this method. The best motivation for using the Lagrangian relaxation for applied optimization was the work of Held and Karp [41] who successfully employed 
this method to solve the traveling salesman problem. Since then the Lagrangian relaxation has been using widely for discrete optimization problems as well as for facility location problems. In UFLP, for example, the common Lagrangian relaxation technique is to relax the assignment constraints. However, in CFLP (capacitated facility location problem) either of the assignment constraints or capacity constraints can be relaxed; see, for instance, [42, 43]. LMRP and CLMRP, which provide a basis for many distribution network design problems, are variants of UFLP and CFLP, respectively. These models can also be solved by relaxing the same constraints that are relaxed in their base model, or any other constraint depending on the mathematical model; see, for instance, $[14,17,25]$.

\section{Problem Definition and Modeling}

This paper aims to design a three-level distribution network for perishable products consisting of one supplier, a set of retailers, and a set of distribution centers (DCs). The DCs order products from the supplier under an EOQ $(Q, r)$ inventory policy and store them to meet the demands of retailers. The EOQ policy determines the order quantity that minimizes total ordering and working inventory costs. However, in $(Q, r)$ policy, when the inventory level drops below the reorder point $(r)$, an order of $Q$ will be placed [44]. In order to approximate the EOQ $(Q, r)$ policy, as discussed by $[14,26,45]$, the order quantity must be determined initially under basic EOQ inventory policy, and then based on the order quantity, the reorder point $(r)$ is calculated.

This paper considers that the demands of retailers are independent and follow a normal distribution. Moreover, the retailers do not hold any inventory, and the inventory of retailers (working inventory and safety stock) is centralized in a number of DCs. This situation provides the system the opportunity of exploiting the advantages of risk pooling that eventually reduces the inventory costs.

A model is developed to determine the configuration and inventory control decision of the network. This model is an extension of the location model with risk pooling (LMRP), which was developed by Daskin et al. [25]. In LMRP, the ordering cycle is calculated by the formula $Q / D$, where $Q$ is the order quantity and $D$ is the annual mean demand of the DCs. However, if products are perishable and their lifetime is less than the period of the ordering cycle, then this inventory policy is not appropriate. This is because, according to Figure 1, before all the products are demanded by the retailers, their lifetimes are over. To avoid this situation, the model should specify a condition on ordering cycle, such that it does not exceed the lifetime, as is shown in Figure 2.

An underlying issue that must be considered regarding perishable products is the dependency of their lifetimes on storage conditions. Ordinarily, any improvement in the storage conditions increases the inventory holding costs, but consequently a longer lifetime is achieved. Therefore, managers of a distribution network have to choose between increasing inventory costs (longer lifetime) and reducing the ordering cycle (shorter lifetime). The model that is



Figure 1: Inventory cycle and lifetime.

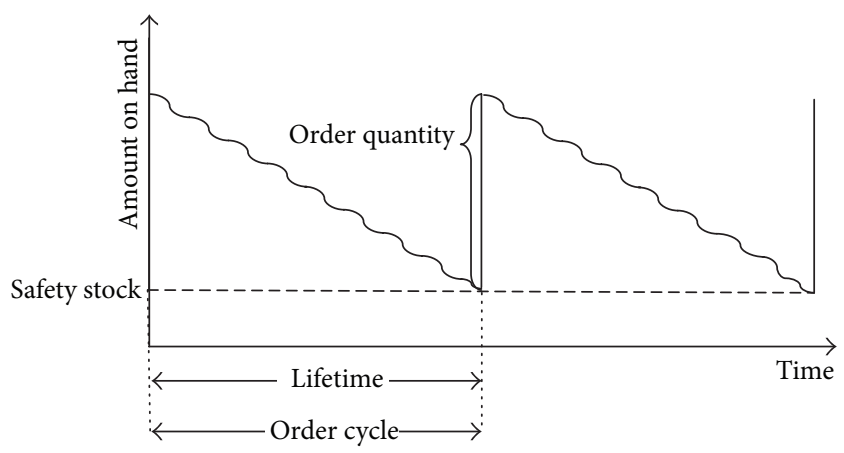

FIGURE 2: Inventory cycle for a perishable product.

developed in this study, in addition to determining the network configuration and inventory control decisions, helps managers calculate such a trade-off.

The remainder of this section describes how the model is formulated. Notation used to model the problem is listed, at the end of the paper.

3.1. Objective Function. This problem is formulated as a nonlinear mixed integer mathematical model. The objective function minimizes the total annual costs, comprising the following: holding inventory and safety stock cost, ordering cost, transportation cost, and fixed installation cost of DCs. The components of the objective function is described in the following.

3.1.1. Holding Cost. The total inventory maintained in the system consists of two components: working inventory and safety stock. The annual working inventory cost for each DC equals the average inventory on hand multiplied by the inventory cost. The safety stock cost is computed by multiplying the amount of safety stock by the inventory cost. If the demands of retailers are independent and follow a normal distribution, the safety stock of $\mathrm{DC}_{i}$, that is, $S S_{i}$, is achieved by the formula $Z_{\alpha} \sqrt{\mathrm{lt}_{i}} \sqrt{V_{i}}$. Symbol $V_{i}$ represents the variance of $\mathrm{DC}_{i}$ that equals the summation of the variances of the retailers' demands that are assigned to that DC. To find $V_{i}$, it is considered at the moment that the assignment of retailers 


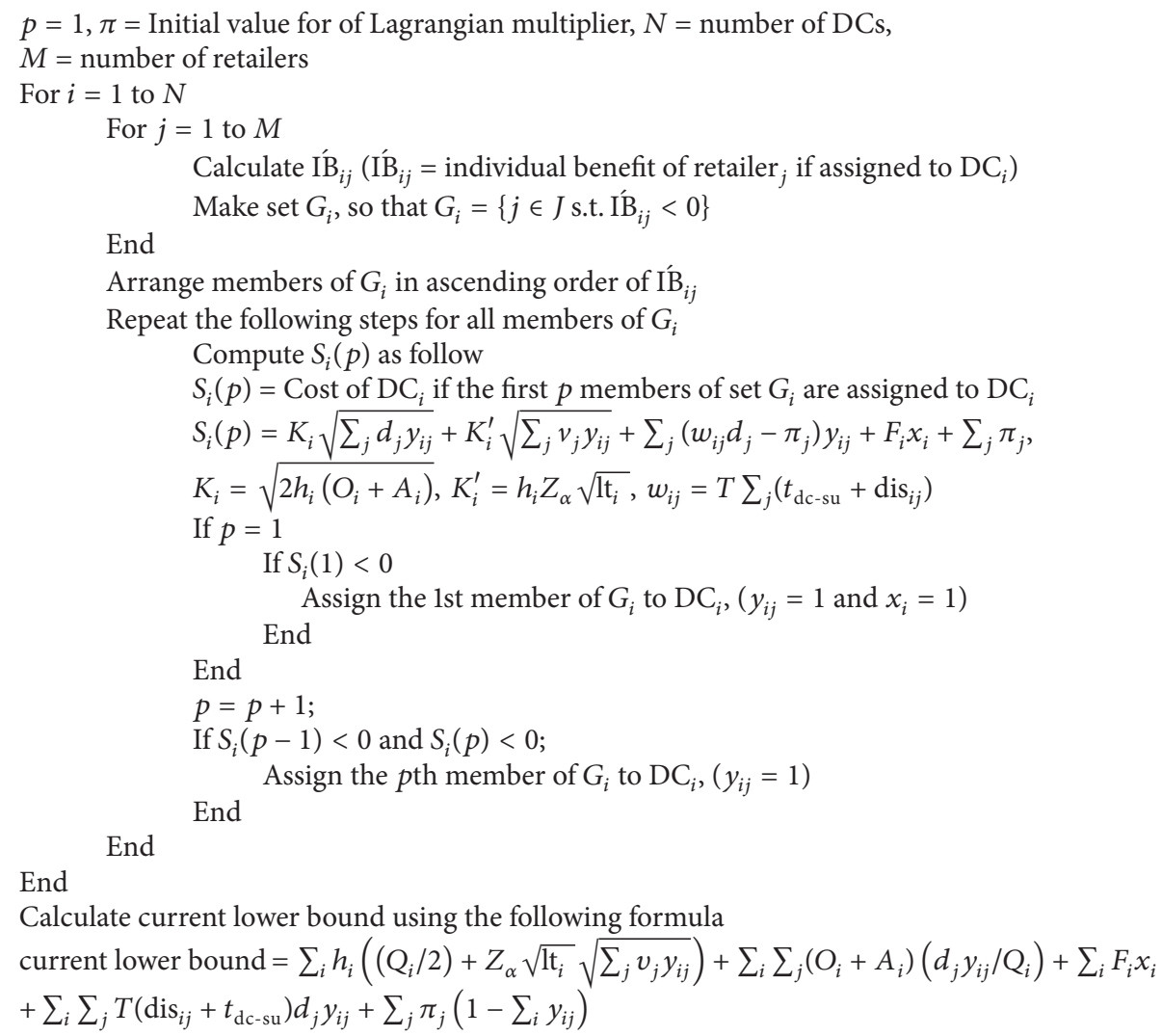

Algorithm 1: Lower bound calculation.

to DCs is known. Therefore, $V_{i}=\sum_{j}\left(v_{j} y_{i j}\right)$ and the inventory cost of $\mathrm{DC}_{i}$ can be written as

$$
\frac{h_{i} Q_{i}}{2}+Z_{\alpha} h_{i} \sqrt{\mathrm{lt}_{i}} \sqrt{\sum_{j}\left(v_{j} y_{i j}\right)} .
$$

In (1), the first term represents the average working inventory holding, cost and the second term is the safety stock holding cost.

3.1.2. Ordering Cost. Ordering cost of $\mathrm{DC}_{i}$ can be formulated as

$$
\frac{O_{i} \sum_{j}\left(d_{j} y_{i j}\right)}{Q_{i}}
$$

where $\sum_{j}\left(d_{j} y_{i j}\right) / Q_{i}$ represents the number of orders placed by $\mathrm{DC}_{i}$ per year.

3.1.3. Transportation Cost. The transportation cost from the supplier to $\mathrm{DC}_{i}$ and from there to the retailers is calculated by (3). In this formula, the first term is the fixed transportation cost that depends on the number of shipments (shipment size is assumed to be equal to $Q$ ), and the second term is the variable transportation cost that depends on the number of items shipped. Therefore,

$$
\frac{A_{i} \sum_{j}\left(d_{j} y_{i j}\right)}{Q_{i}}+T \sum_{j} d_{j} y_{i j}\left(\operatorname{dis}_{i j}+t_{\mathrm{dc}-\mathrm{su}}\right) \quad \forall i \in I .
$$

3.1.4. Fixed Setup Cost. The cost of establishing $\mathrm{DC}_{i}$ is calculated by the following:

$$
F_{i} x_{i} \quad \forall i \in I,
$$

where $x_{i}$ is a binary variable that is equal to 1 if $\mathrm{DC}_{i}$ is established; otherwise, it is equal to 0 .

3.1.5. Effect of Lifetime on the Inventory Policy. If the products' deterioration begins after they are released from the supplier, then upon delivery to the distribution centers, they will have lost part of their lifetime equivalent to the lead time. On the other hand, according to Figure 3, the maximum time that a product remains in a DC is equal to the ordering cycle plus $t_{s s}$. The period $t_{s s}$ is the required time to replace the safety stock by a new inventory.

Therefore we can write

$$
\frac{Q_{i}}{D_{i}}+\frac{S S_{i}}{D_{i}} \leq \mathrm{pt}_{i}-1 \mathrm{t}_{i}
$$


where the first term represents the order cycle and the second term represents $t_{s s}$. This inequality can be rewritten as follows:

$$
Q_{i} \leq(\mathrm{pt}-\mathrm{lt}) D_{i}-S S_{i}
$$

Substituting $D_{i}$ and $S S_{i}$ by their amounts into the above inequality, the following constraint for order quantity is achieved:

$$
Q_{i} \leq\left(\mathrm{pt}_{i}-\mathrm{lt}_{i}\right) \sum_{j}\left(d_{j} y_{i j}\right)-Z_{\alpha} \sqrt{\mathrm{lt}_{i}} \sqrt{\sum_{j}\left(v_{j} y_{i j}\right)} \quad \forall i \in I .
$$

3.1.6. Total Annual Cost. According to the components of cost described above, the total annual costs can be written as

$$
\begin{aligned}
\min & \sum_{i} h_{i}\left(\frac{Q_{i}}{2}+Z_{\alpha} \sqrt{\mathrm{lt}_{i}} \sqrt{\sum_{j}\left(v_{j} y_{i j}\right)}\right) \\
+ & \sum_{i} \sum_{j}\left(O_{i}+A_{i}\right) \frac{\left(d_{j} y_{i j}\right)}{Q_{i}}+\sum_{i} F_{i} x_{i} \\
+ & \sum_{i} \sum_{j} T\left(\operatorname{dis}_{i j}+t_{\mathrm{dc}-\mathrm{su}}\right) d_{j} y_{i j}
\end{aligned}
$$

s.t.

$$
\begin{gathered}
\sum_{i} y_{i j}=1 \quad \forall j \in J \\
x_{i} \geq y_{i j}, \quad \forall i \in I, \forall j \in J \\
Q_{i} \leq\left(\mathrm{pt}_{i}-\mathrm{lt}_{i}\right) \sum_{j}\left(d_{j} y_{i j}\right)-Z_{\alpha} \sqrt{\mathrm{lt}_{i}} \sqrt{\sum_{j}\left(v_{j} y_{i j}\right)}, \quad \forall i \in I, \\
x_{i}, y_{i j}=\{1,0\}, \quad \forall i \in I, \forall j \in J .
\end{gathered}
$$

Constraint (9) ensures a single-sourcing strategy for retailers. Constraint (10) makes sure that retailers are not assigned to nonestablished DCs. Constraint set (11) avoids the products from remaining in each DC for longer than their lifetime, and constraint set (12) specifies that $x_{i}, y_{i j}$ are binary variables.

\section{Solution Method}

To solve the model, a heuristic Lagrangian relaxation algorithm is developed. The Lagrangian relaxation is one of the most widely used techniques that have been applied successfully to solve distribution network design problems. In the Lagrangian relaxation, the constraints that introduce difficulty to the problem are removed and added to the objective function with a penalty term. The new problem provides a lower (upper) bound for the main minimization (maximization) problem [46]. Solution quality and high speed are two significant specifications of this method, as reported in studies by Daskin et al. [25], Miranda and Garrido
[17], Shen [27], Miranda and Garrido [37], Sourirajan et al. [29], Max Shen and Qi [16], Snyder et al. [47], Qi and Shen [31], Miranda and Garrido [7], Ozsen et al. [14], Mak and Shen [48], and Park et al. [45]. In the following, the procedure of finding upper and lower bounds on the optimal value of the proposed model are described.

4.1. Lower Bound. As the objective function (8) subject to (9)-(12) is an extension of UFLP, to find a lower bound, the DC retailer assignment constraint (9) is relaxed. The new function is called a Lagrangian dual problem, as is shown by (13) subject to (14)-(16). Lagrangian dual problem provides a lower bound for the main objective function (8) subject to (9)-(12), as follows:

$$
\begin{aligned}
\max _{\gamma \geq 0} \min _{x, y} \sum_{i} h_{i}\left(\frac{Q_{i}}{2}+Z_{\alpha} \sqrt{\mathrm{lt}_{i}} \sqrt{\sum_{j} v_{j} y_{i j}}\right) \\
+\sum_{i} \sum_{j}\left(O_{i}+A_{i}\right) \frac{d_{j} y_{i j}}{Q_{i}}+\sum_{i} F_{i} x_{i} \\
+\sum_{i} \sum_{j} T\left(\operatorname{dis}_{i j}+t_{\mathrm{dc}-\mathrm{su}}\right) d_{j} y_{i j}+\sum_{j} \pi_{j}\left(1-\sum_{i} y_{i j}\right)
\end{aligned}
$$

s.t.

$$
\begin{gathered}
x_{i} \geq y_{i j} \quad \forall i \in I, \forall j \in J, \\
Q_{i} \leq\left(\mathrm{pt}_{i}-\mathrm{lt}_{i}\right) \sum_{j}\left(d_{j} y_{i j}\right)-Z_{\alpha} \sqrt{\mathrm{lt}_{i}} \sqrt{\sum_{j}\left(v_{j} y_{i j}\right)} \quad \forall i \in I, \\
x_{i}, y_{i j}=\{1,0\} \quad \forall i \in I, \forall j \in J .
\end{gathered}
$$

To solve the objective function (13) subject to (14)-(16), it is considered at the moment that constraint set (15) is not active. Therefore, the order quantity $Q_{i}$ is calculated by the following formula:

$$
Q_{i}=\sqrt{\frac{2\left(O_{i}+A_{i}\right) \sum_{j} d_{j} y_{i j}}{h_{i}} .}
$$

By substituting $Q_{i}$ in formula (13) the following function is obtained:

$$
\begin{aligned}
\max _{\gamma \geq 0} \min _{x, y} \sum_{i} K_{i} \sqrt{\sum_{j} d_{j} y_{i j}}+\sum_{i} K_{i}^{\prime} \sqrt{\sum_{j} v_{j} y_{i j}} \\
+\sum_{i} \sum_{j}\left(w_{i j} d_{j}-\pi_{j}\right) y_{i j}+\sum_{i} F_{i} x_{i}+\sum_{j} \pi_{j},
\end{aligned}
$$

where

$$
\begin{gathered}
K_{i}=\sqrt{2 h_{i}\left(O_{i}+A_{i}\right)}, \quad K_{i}^{\prime}=h_{i} Z_{\alpha} \sqrt{\mathrm{lt}_{i}}, \\
w_{i j}=T \sum_{j}\left(t_{\mathrm{dc}-\mathrm{su}}+\operatorname{dis}_{i j}\right) .
\end{gathered}
$$






Algorithm 2: Upper bound calculation.

Objective function (18) is then decomposed into subproblems for each DC candidate location, as follows:

$$
\begin{aligned}
S_{i}=\min K_{i} \sqrt{\sum_{j} d_{j} y_{i j}}+K_{i}^{\prime} \sqrt{\sum_{j} v_{j} y_{i j}} \\
+\sum_{j}\left(w_{i j} d_{j}-\pi_{j}\right) y_{i j}+F_{i} x_{i}+\sum_{j} \pi_{j} .
\end{aligned}
$$

The KKT conditions for the problem are

$$
\begin{aligned}
\frac{\partial L_{i}(y, \pi)}{\partial y_{i j}}= & \frac{\sum_{i} K_{i} \sqrt{\sum_{j} d_{j} y_{i j}}+\sum_{i} K_{i}^{\prime} \sqrt{\sum_{j} v_{j} y_{i j}}}{\partial y_{i j}} \\
& +\sum_{i} \sum_{j}\left(w_{i j} d_{j}-\pi_{j}\right)=0 \quad \forall j \in J,
\end{aligned}
$$

$$
\begin{gathered}
\pi_{j} \geq 0, \quad \forall j \in J, \\
\sum_{i} y_{i j}-1=0, \quad \forall j \in J .
\end{gathered}
$$

The first term in (21) is called the marginal inventory cost of retailer ${ }_{j}$, that is the difference in the inventory cost of $\mathrm{DC}_{i}$ between assigning retailer ${ }_{j}$ to $\mathrm{DC}_{i}$ or not. Let the symbol $m_{i j}$ represent the marginal inventory cost of retailer ${ }_{j}$. Then according to $S_{i}, m_{i j}$ can be written as in the following:

$$
\begin{aligned}
m_{i j}= & K_{i}\left(\sqrt{\sum_{j} d_{j} y_{i, j}}-\sqrt{\sum_{j-1} d_{j-1} y_{i, j-1}}\right) \\
& +K_{i}^{\prime}\left(\sqrt{\sum_{j} v_{j} y_{i, j}}-\sqrt{\sum_{j-1} v_{j-1} y_{i, j-1}}\right) .
\end{aligned}
$$






Algorithm 3: Lagrangian relaxation heuristic algorithm.

To make this function independent from other retailers assigned to the same $\mathrm{DC}_{i}$, a lower bound of it is selected to work with, as follows:

$$
m_{i j}=K_{i} \log d_{j}+K_{i}^{\prime} \log v_{j} .
$$

So if retailer ${ }_{j}$ is assigned to $\mathrm{DC}_{i}$, the individual benefit of it would be as follows:

$$
\mathrm{IB}_{i j}=K_{i} \log d_{j}+K_{i}^{\prime} \log v_{j}+w_{i, j} d_{j}-\pi_{j} .
$$

If $\mathrm{IB}_{i j}>0$, then retailer ${ }_{j}$ cannot be assigned to $\mathrm{DC}_{i}$, and therefore, $y_{i j}=0$ for all retailer. However, if $\mathrm{IB}_{i j} \leq 0$, then retailer ${ }_{j}$ will be assigned to $\mathrm{DC}_{i}$ if it leads to a negative value for $S_{i}$. Therefore, for each DC, initially a list of retailers having the necessary condition of $\mathrm{IB}_{i j} \leq 0$ is made. Then, set $G_{i}$ is made as follow by arranging retailers in ascending order of their $\mathrm{IB}_{i j}$ :

$$
G_{i}=\left\{j \in J \text { s.t. } \mathrm{IB}_{i j-1} \leq \mathrm{IB}_{i j}, \mathrm{IB}_{j} \leq 0\right\} .
$$

The first retailer from set $G_{i}$ is assigned to $\mathrm{DC}_{i}$ if it leads to a negative value for $S_{i}$. Each of the following retailers is assigned one by one to $\mathrm{DC}_{i}$ if the previous retailer (from set $G_{i}$ ) is assigned to $\mathrm{DC}_{i}$ and if its assignment to $\mathrm{DC}_{i}$ leads to a better (lower) value for $S_{i}$. Retailers that are assigned to $\mathrm{DC}_{i}$ are removed from set $G_{i}$ and are added to set $G_{i}$. If there is at least one retailer in $G_{i}$, then $x_{i}$ is set to 1 . For all retailers that belong to set $G_{i}, y_{i j}$ is set to 1 .
Moreover, the value of the Lagrangian multiplier $\pi_{j}$ in each iteration of the algorithm is updated using the subgradient optimization technique. The lower-bound calculation steps are presented in Algorithm 1.

4.2. Upper Bound. The solution that is found by the lower bound might be infeasible. Therefore, the upper bound modifies it to be a feasible solution for the main objective function. To achieve this, in the developed algorithm of this paper, at first, the lower bound solution is displayed in the form of a 0-1 matrix. The number of rows of this matrix is equal to the number of DCs, and the number of columns is equal to the number of retailers. If the array of $i$ th row and $j$ th column equals 1 , it means that retailer ${ }_{j}$ is assigned to $\mathrm{DC}_{i}$. The lower-bound solution described in Section 4.1 may need to be modified in two steps: the first step takes into account the single-sourcing constraint and the next step considers constraint (11) that is also referred to as $Q$ constraint in this text. To do the first step, the retailers that are assigned to no DC are considered, and all the arrays of their corresponding columns are initially set to 1 . Then, for each DC (row) the objective function is calculated. The DC that has the minimum objective function is selected and all of its arrays are set to be fixed. Then, if retailers of this DC are also assigned to other DCs, all other similar assignments are removed. This procedure is repeated until all retailers are allocated to only one DC. To do the second step, a DC that its $Q$ constraint is violated is selected. Then its retailers are removed one by one until its $Q$ constraint is satisfied. The first retailer that would be removed is the one 


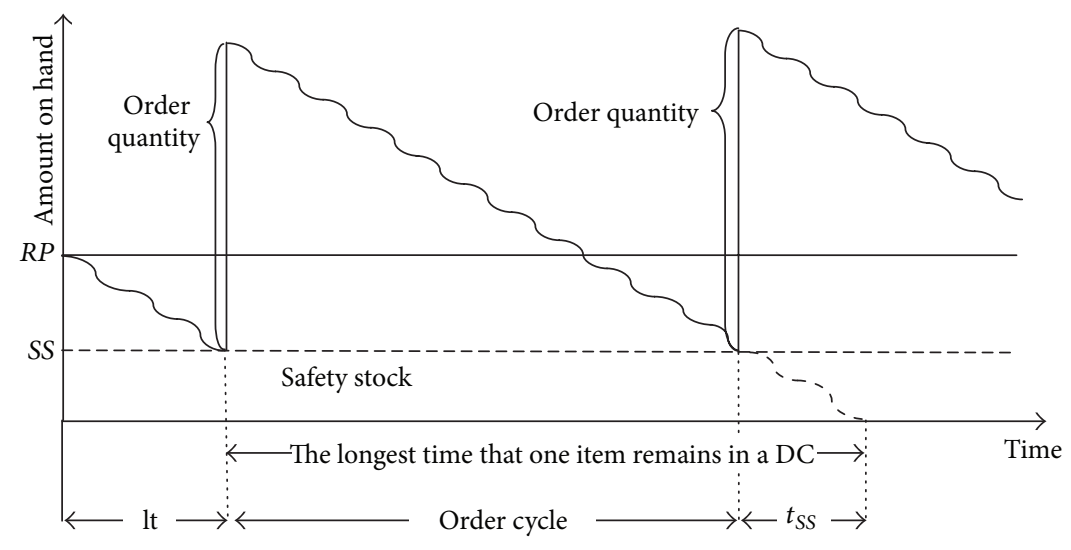

FIGURE 3: Profile of inventory level over time.

that was assigned last to set $G_{i}$ in lower-bound calculation. The removed retailer is assigned to another DC that increases the total cost the least, considering feasibility conditions. The upper bound calculation steps and the Lagrangian relaxation algorithm are presented in Algorithms 2 and 3, respectively.

4.3. Procedure of Computing Q. To obtain the value of order quantity $Q_{i}$, as mentioned before, at first the derivative of the objective function with respect to $Q_{i}$ is calculated and is solved for $Q_{i}$, as follows:

$$
Q_{i}=\sqrt{\frac{2\left(A_{i}+O_{i}\right) D_{i}}{h_{i}}} \quad \forall i \in I .
$$

If $Q_{i}$ violates constraint (11), then $Q_{i}$ will change to the maximum amount that constraint (11) forces it to be, as is written in the following:

$$
Q_{i}=\left(\mathrm{pt}_{i}-\mathrm{lt}_{i}\right) \sum_{j}\left(d_{j} y_{i j}\right)-Z_{\alpha} \sqrt{\mathrm{lt}_{i}} \sqrt{\sum_{j}\left(v_{j} y_{i j}\right)} .
$$

\section{Computational Results and Discussion}

The computational results are divided into three parts. The first part is to validate the model and heuristic algorithm. The second part is to investigate the performance of the algorithm, and the last part provides some examples to demonstrate the main application of the model. For the first and second parts, the model and algorithm are tested on 15node and 49-node data sets derived from [49]. For the last part, along with 15-node and 49-node data sets, 88-node data set is also considered. Each node in each data set represents a retailer. A number of retailers must be selected to serve as distribution centers. In this study, the means and variances of retailers' demands are selected to be the same as the demand parameters of [49]. Distances between retailers are calculated using the great circle distance formula, based on the longitude and latitude of retailers' locations. Fixed installation costs are set to the fixed installation costs, as considered by [49], but multiplied by 10 . Variable transportation costs are set
TABLE 1: Parameter of the Lagrangian relaxation.

\begin{tabular}{lc}
\hline Maximum number of iterations & 1500 \\
Number of nonimproving iterations before halving & 30 \\
step size & 2 \\
Initial value of step size & $10^{-7}$ \\
Minimum value of step size & $10(\bar{d}+\bar{f})$ \\
Initial value of the Lagrangian multiplier & $0.1 \%$ \\
Maximum optimality gap ((UB - LB) /UB $) * 100 \%$ & \\
\hline
\end{tabular}

to 50 units of cost. Lead time is set to 1 day, and the sum of fixed ordering and transportation costs is set to 100 units of cost. As this paper is motivated by a platelet blood distribution network, inventory holding costs are derived from the work of [50], which studied the inventory control of blood platelets. The parameters of Lagrangian relaxation method are presented in Table 1 .

In Table $1, \bar{d}, \bar{f}$ are the average demands of the retailers and the average fixed installation costs of the DCs, respectively. The problem is written in $\mathrm{C}++$, and the results are obtained on a T2350, 1.86 GHZ with 1 GB RAM.

5.1. Model and Algorithm Validation. The model and heuristic algorithm are validated using sensitivity analysis. The sensitivity analysis is performed on key parameters, including variances of demands, inventory cost, fixed facility installation costs, and lifetimes of commodities. The value of the lifetime varies between 3 and 9 days and the other parameters are varies between $30 \%$ and $-30 \%$ of their actual value.

Figures 4 and 5 show changes in the objective function in terms of the lifetime for the 15-node and 49-node data sets, respectively. Each point in this curve is the average of $7^{3}(=$ 343) instances that are made by changing the variances of demand, inventory holding costs, and fixed installation costs at seven levels of $\pm 30 \%, \pm 20 \%, \pm 10 \%$, and $0 \%$.

As is expected, the value of the objective function decreases as the lifetime gets longer. The numbers written above the curves in Figures 4 and 5 and also Figures 6-11 


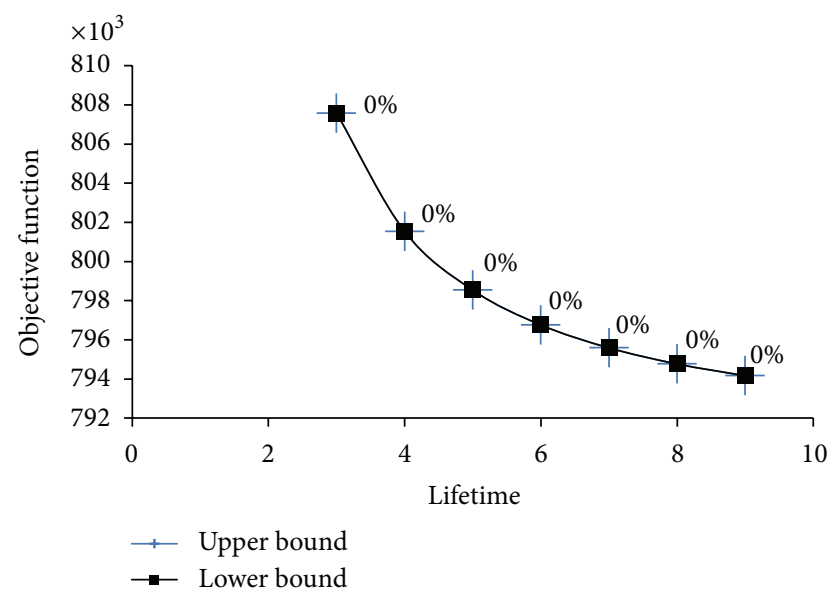

FIGURE 4: Sensitivity of objective function to changes in the lifetime for 15-node data set.



FIGURE 5: Sensitivity of objective function to changes in the lifetime for 49-node data set.

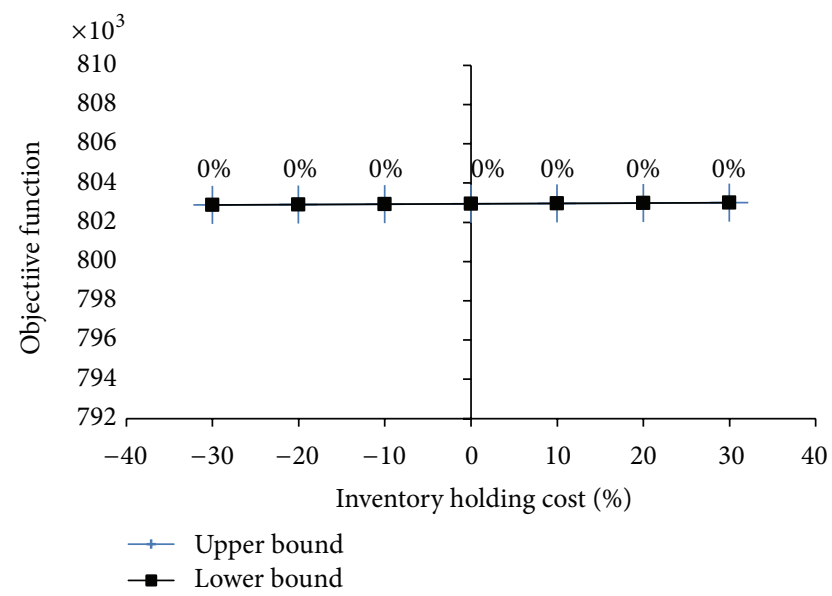

FIGURE 6: Sensitivity of objective function to changes in inventory cost for 15-node date set.

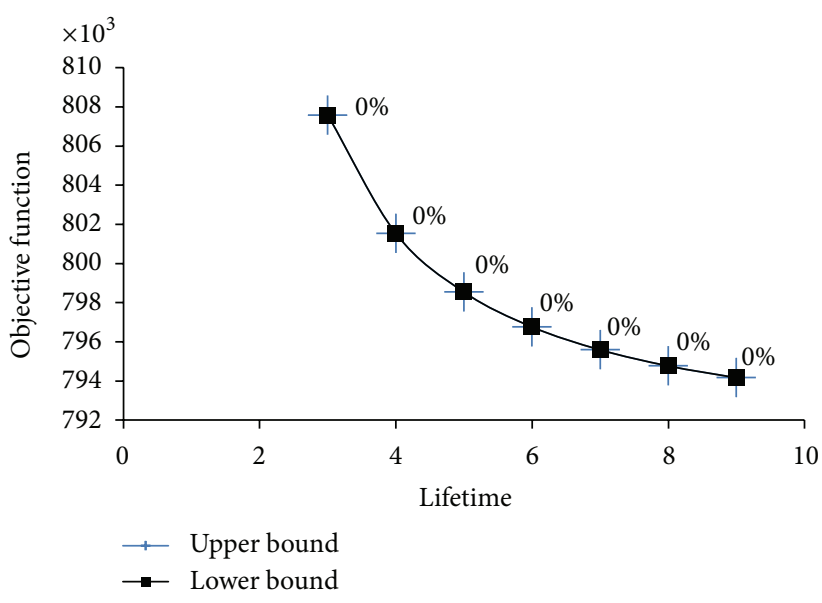

FIGURE 7: Sensitivity of objective function to changes in inventory cost for 49-node date set.

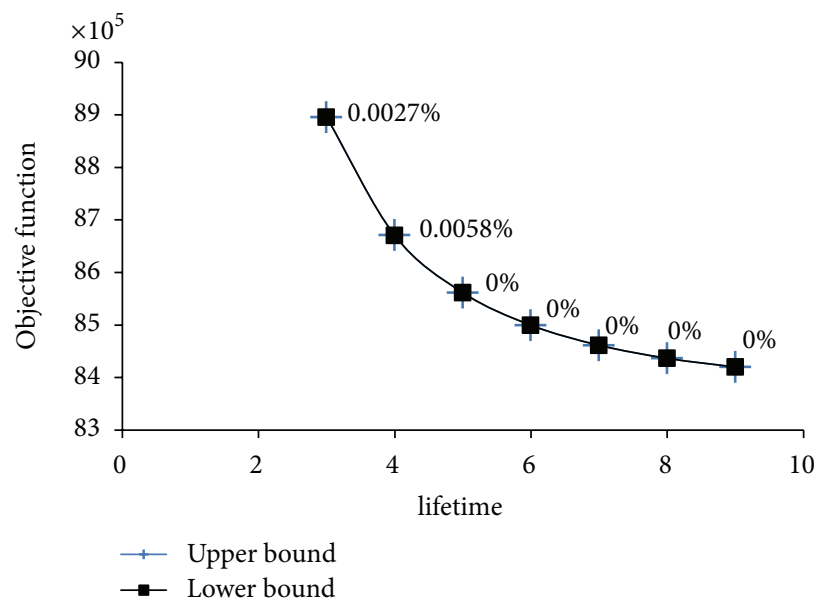

FIGURE 8: Sensitivity of objective function to changes in variances of demands for 15-node data set.



FIGURE 9: Sensitivity of objective function to changes in variances of demands for 49-node data set. 


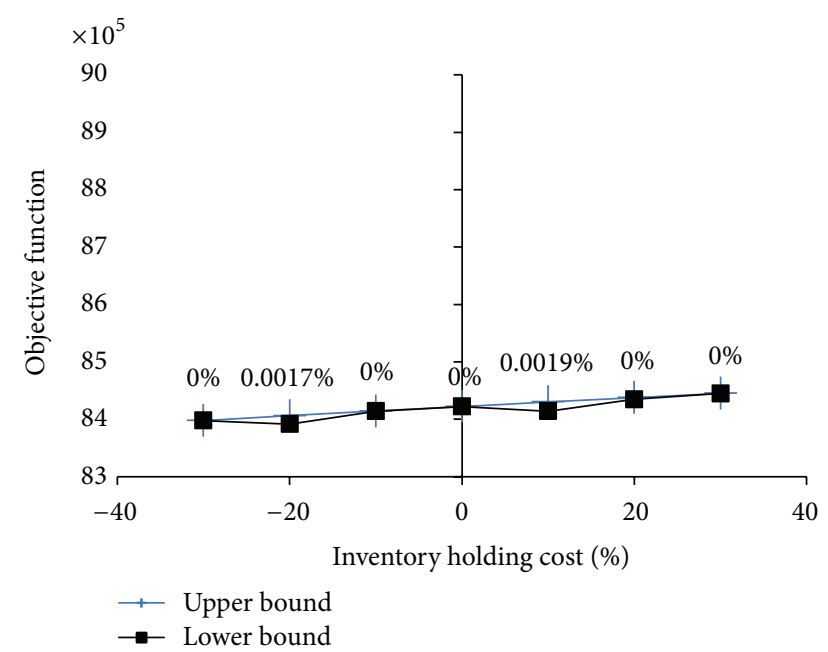

FIGURE 10: Sensitivity of objective function to changes in fixed installation cost for 15-node data sets.



FIGURE 11: Sensitivity of objective function to changes in fixed installation cost for 49-node data sets.

TABLE 2: Algorithm performance.

\begin{tabular}{lccc}
\hline Data set & $\begin{array}{c}\text { Average CPU } \\
\text { time (second) }\end{array}$ & $\begin{array}{c}\text { Average number of } \\
\text { iterations }\end{array}$ & $\begin{array}{c}\text { Average } \\
\text { optimality gap }\end{array}$ \\
\hline 15-node & 0.027 & 11 & 0 \\
49-node & 0.8 & 98 & $0.07 \%$ \\
\hline
\end{tabular}

TABLE 3: Lifetime and inventory holding cost of blood platelet driven from [50].

\begin{tabular}{lccc}
\hline Parameters & Alternative 1 & Alternative 2 & Alternative 3 \\
\hline Lifetime & 4 & 5 & 6 \\
Inventory cost & 0.2995 & 0.4947 & 0.6928 \\
\hline
\end{tabular}

display the average optimality gaps. The Optimality gap represents the maximum gap between the optimal solution and the solutions found by the Lagrangian relaxation algorithm. The optimality gap is computed as follows:

$$
\text { Optimality gap }=\frac{(\text { Upper bound }- \text { lower bound })}{\text { Upper bound }} \times 100 \text {. }
$$

Figures 6 and 7 display the variation of the objective function versus changes in inventory holding costs for the 15- and 49node data sets, respectively. Both curves are ascending, but it is not very clear, especially when they are compared with changes of the objective function versus the lifetime. Figures 8 and 9 also show the variation of the objective function, but against changes in variance of demand. Despite variance changes within a wide range, a very slight increase is observed in the value of the objective function. The most influential parameter on the objective function is the fixed installation cost, as shown by Figures 10 and 11. If these curves had been presented on a graph with the same scale as the previous graphs, only a small part of the curve could be displayed. Therefore, the scales of the vertical axes of these two graphs are different. Figures 10 and 11 show that significant changes occur in the objective function when the fixed costs change.

5.2. Performance of the Algorithm. To show the performance of the algorithm in terms of CPU time, number of iterations required to solve each problem, and the optimality gap, the averages of these values are computed and presented in Table 2. Each number in Table 2 is the average of corresponding values obtained by running the algorithm for $9604(=343 \times 7 \times 4)$ times in Section 5.1, where 4 is the number of input parameters which are selected for sensitivity analysis and 7 is the number of times each parameter has been changed.

Table 2 shows that the average optimality gaps are small enough to say that the algorithm finds optimal or near optimal solutions. Moreover, the average CPU time and the average number of iterations that the algorithm needs to find the solution imply that the algorithm is fast.

5.3. Application of the Algorithm. The main application of the model of this paper is to provide a trade-off between selecting longer lifetime (increasing inventory cost) and reducing the ordering cycle (shorter lifetime). If the product to be distributed is blood platelets, according to [50], three alternatives for storage conditions exist. These alternatives are shown in Table 3. For instance, alternative 1 represents a storage condition in which the product remains safe for up to four days, and the inventory holding cost is 0.2995 units of cost.

The model is solved for the 15-node, 49-node, and 88node data sets taken from [49], and the results are displayed in Table 4. The last column of the table demonstrates the alternative that is selected in terms of the objective function. For example, for the 15-node data set, alternative 3 is the best despite its highest inventory cost. However, for the 88-node case, the lowest inventory cost alternative is optimal. 
TABLE 4: Value of objective function corresponding to different alternatives.

\begin{tabular}{lcccc}
\hline Data set & Alternative 1 & Alternative 2 & Alternative 3 & Best alternative \\
\hline 15 -node & 1779198 & 1776691 & 1775583 & 3 \\
49 -node & 14257158 & 14209680 & 14226085 & 2 \\
88 -node & 52124780 & 52149816 & 52215864 & 1 \\
\hline
\end{tabular}

\section{Conclusion}

Perishable products comprise a large proportion of products that are transferred daily from suppliers to the customers. However, studies on the distribution network design of perishable products are rare. This study extended the LMRP by considering the lifetime of the product that is being distributed. The developed model determines the optimal configuration and the inventory control decisions of the network. In addition, the model develops a trade-off between enhancing storage conditions, interpreted as higher inventory costs but longer lifetime, and accepting less inventory costs but having a product with a shorter lifetime. Sensitivity analysis on key parameters is performed to validate the model and solution method. For future research, it is suggested to incorporate into the DND model an inventory control policy of those perishable products whose value declines with time.

\section{Notation}

Sets

$J$ : Set of retailers

I: Set of candidate DC locations.

Indices

$i$ : Index for DCs

$j$ : Index for retailers.

\section{Input Parameters}

$F_{i}: \quad$ Annual fixed setup cost for $\mathrm{DC}_{i}$

$T$ : $\quad$ Transportation cost per unit of product per unit of distance

$t_{\mathrm{dc}-\mathrm{su}}$ : Per item transportation cost from the supplier to a DC

$A_{i}$ : $\quad$ Per shipment transportation cost from supplier to $\mathrm{DC}_{i}$

$h_{i}$ : Inventory holding cost at $\mathrm{DC}_{i}$ per unit of product per year

$\mathrm{O}_{i}$ : $\quad$ Fixed ordering cost per order placed by $\mathrm{DC}_{i}$ to the supplier

$d_{j}$ : $\quad$ Annual mean demand of retailer ${ }_{j}$

$D_{i}$ : Annual mean demand of $\mathrm{DC}_{i}$

$v_{j}$ : $\quad$ Variance of annual demand for retailer ${ }_{j}$

$V_{i}$ : Variance of annual demand for $\mathrm{DC}_{i}$

dis $_{i j}$ : Distance between $\mathrm{DC}_{i}$ and retailer ${ }_{j}$ $\mathrm{lt}_{i}$ : Lead time in terms of year from the supplier to $\mathrm{DC}_{i}$

pt: Lifetime of products

$\alpha$ : Level of service that has to be achieved at the retailers

$Z_{\alpha}$ : Standard normal deviate such that $P\left(z \leq z_{\alpha}\right)=\alpha$.

\section{Decision Variables}

$\mathrm{Q}_{i}$ : Order quantity of $\mathrm{DC}_{i}$

$y_{i j}$ : Binary variable, taking the value 1 if retailer ${ }_{j}$ is assigned to $\mathrm{DC}_{i}$ and 0 otherwise

$x_{i}$ : Binary variable, taking the value 1 if $\mathrm{DC}_{i}$ is open and 0 otherwise.

\section{References}

[1] M. Ferguson and M. E. Ketzenberg, "Information sharing to improve retail product freshness of perishables," Production and Operations Management, vol. 15, no. 1, pp. 57-73, 2006.

[2] Who, Global Database on Blood Safety, Summary Report 2011, World Health Organization, 2011, http://www.who.int/bloodsafety/global_database/GDBS_Summary_Report_2011.pdf.

[3] P. Amorim, H. Meyr, C. Almeder, and B. Almada-Lobo, "Managing perishability in production-distribution planning: a discussion and review," Flexible Services and Manufacturing Journal, pp. 1-25, 2011.

[4] C. Kouki, E. Sahin, Z. Jemaï, and Y. Dallery, "Assessing the impact of perishability and the use of time temperature technologies on inventory management," International Journal of Production Economics, 2010.

[5] S. Minner and S. Transchel, "Periodic review inventory-control for perishable products under service-level constraints," $O R$ Spectrum, vol. 32, no. 4, pp. 979-996, 2010.

[6] J. Jia and Q. Hu, "Dynamic ordering and pricing for a perishable goods supply chain," Computers \& Industrial Engineering, vol. 60, no. 2, pp. 302-309, 2011.

[7] P. A. Miranda and R. A. Garrido, "Valid inequalities for Lagrangian relaxation in an inventory location problem with stochastic capacity," Transportation Research Part E: Logistics and Transportation Review, vol. 44, no. 1, pp. 47-65, 2008.

[8] P. A. Miranda and R. A. Garrido, "Inventory service-level optimization within distribution network design problem," International Journal of Production Economics, vol. 122, no. 1, pp. 276-285, 2009.

[9] F. Altiparmak, M. Gen, L. Lin, and T. Paksoy, "A genetic algorithm approach for multi-objective optimization of supply 
chain networks," Computers and Industrial Engineering, vol. 51, no. 1, pp. 196-215, 2006.

[10] M. Faccio, A. Persona, F. Sgarbossa, and G. Zanin, "Multi-stage supply network design in case of reverse flows: a closed-loop approach," International Journal of Operational Research, vol. 12, no. 2, pp. 157-191, 2011.

[11] A. Amiri, "Designing a distribution network in a supply chain system: formulation and efficient solution procedure," European Journal of Operational Research, vol. 171, no. 2, pp. 567-576, 2006.

[12] S. Melkote and M. S. Daskin, "Capacitated facility location/ network design problems," European Journal of Operational Research, vol. 129, no. 3, pp. 481-495, 2001.

[13] M. Punakivi and V. Hinkka, "Selection criteria of transportation mode: a case study in four finnish industry sectors," Transport Reviews, vol. 26, no. 2, pp. 207-219, 2006.

[14] L. Ozsen, C. R. Coullard, and M. S. Daskin, "Capacitated warehouse location model with risk pooling," Naval Research Logistics, vol. 55, no. 4, pp. 295-312, 2008.

[15] Z. Firoozi, S. Tang, S. Ariafar, and M. K. Ariffin, "An optimization approach for A joint location inventory model considering quantity discount policy," Arabian Journal for Science and Engineering, vol. 38, no. 4, pp. 983-991, 2013.

[16] Z. J. Max Shen and L. Qi, "Incorporating inventory and routing costs in strategic location models," European Journal of Operational Research, vol. 179, no. 2, pp. 372-389, 2007.

[17] P. A. Miranda and R. A. Garrido, "Incorporating inventory control decisions into a strategic distribution network design model with stochastic demand," Transportation Research Part E: Logistics and Transportation Review, vol. 40, no. 3, pp. 183-207, 2004.

[18] O. Berman, D. Krass, and M. M. Tajbakhsh, "A coordinated location-inventory model," European Journal of Operational Research, vol. 217, no. 3, pp. 500-508, 2012.

[19] Y. C. Tsao, D. Mangotra, J. C. Lu, and M. Dong, "A continuous approximation approach for the integrated facility-inventory allocation problem," European Journal of Operational Research, vol. 222, no. 2, pp. 216-228, 2012.

[20] C. I. Hsu, S. F. Hung, and H. C. Li, "Vehicle routing problem with time-windows for perishable food delivery," Journal of Food Engineering, vol. 80, no. 2, pp. 465-475, 2007.

[21] R. A. C. M. Broekmeulen and K. H. van Donselaar, "A heuristic to manage perishable inventory with batch ordering, positive lead-times, and time-varying demand," Computers and Operations Research, vol. 36, no. 11, pp. 3013-3018, 2009.

[22] F. Olsson and P. Tydesjö, "Inventory problems with perishable items: fixed lifetimes and backlogging," European Journal of Operational Research, vol. 202, no. 1, pp. 131-137, 2010.

[23] F. Dabbene, P. Gay, and N. Sacco, "Optimisation of fresh-food supply chains in uncertain environments, part II: a case study," Biosystems Engineering, vol. 99, no. 3, pp. 360-371, 2008.

[24] Z. J. M. Shen, "Integrated supply chain design models: a survey and future research directions," Journal of Industrial and Management Optimization, vol. 3, no. 1, pp. 1-27, 2007.

[25] M. S. Daskin, C. R. Coullard, and Z.-J. M. Shen, "An inventorylocation model: formulation, solution algorithm and computational results," Annals of Operations Research, vol. 110, no. 1-4, pp. 83-106, 2002.

[26] Z. J. M. Shen, C. Coullard, and M. S. Daskin, "A joint locationinventory model," Transportation Science, vol. 37, no. 1, pp. 4055, 2003.
[27] Z. J. M. Shen, "A multi-commodity supply chain design problem," IIE Transactions (Institute of Industrial Engineers), vol. 37, no. 8, pp. 753-762, 2005.

[28] J. Shu, C. P. Teo, and Z. J. M. Shen, "Stochastic transportationinventory network design problem," Operations Research, vol. 53, no. 1, pp. 48-60, 2005.

[29] K. Sourirajan, L. Ozsen, and R. Uzsoy, "A single-product network design model with lead time and safety stock considerations," IIE Transactions (Institute of Industrial Engineers), vol. 39, no. 5, pp. 411-424, 2007.

[30] K. Sourirajan, L. Ozsen, and R. Uzsoy, "A genetic algorithm for a single product network design model with lead time and safety stock considerations," European Journal of Operational Research, vol. 197, no. 2, pp. 599-608, 2009.

[31] L. Qi and Z. J. M. Shen, "A supply chain design model with unreliable supply," Naval Research Logistics, vol. 54, no. 8, pp. 829-844, 2007.

[32] E. Gebennini, R. Gamberini, and R. Manzini, "An integrated production-distribution model for the dynamic location and allocation problem with safety stock optimization," International Journal of Production Economics, vol. 122, no. 1, pp. 286304, 2009.

[33] A. Jha, K. Somani, M. K. Tiwari, F. T. S. Chan, and K. J. Fernandes, "Minimizing transportation cost of a joint inventory location model using modified adaptive differential evolution algorithm," International Journal of Advanced Manufacturing Technology, vol. 60, pp. 1329-4341, 2012.

[34] M. T. Melo, S. Nickel, and F. Saldanha-da-Gama, "A tabu search heuristic for redesigning a multi-echelon supply chain network over a planning horizon," International Journal of Production Economics, vol. 136, no. 1, pp. 218-230, 2012.

[35] H. Shavandi and B. Bozorgi, "Developing a location-inventory model under fuzzy environment," International Journal of Advanced Manufacturing Technology, pp. 1-10, 2012.

[36] A. Atamtürk, G. Berenguer, and Z.-J. Shen, "A conic integer programming approach to stochastic joint location-inventory problems," Operations Research, vol. 60, no. 2, pp. 366-381, 2012.

[37] P. A. Miranda and R. A. Garrido, "A simultaneous inventory control and facility location model with stochastic capacity constraints," Networks and Spatial Economics, vol. 6, no. 1, pp. 39-53, 2006.

[38] S. K. Goyal and B. C. Giri, "Recent trends in modeling of deteriorating inventory," European Journal of Operational Research, vol. 134, no. 1, pp. 1-16, 2001.

[39] K. Kanchanasuntorn and A. Techanitisawad, "An approximate periodic model for fixed-life perishable products in a twoechelon inventory-distribution system," International Journal of Production Economics, vol. 100, no. 1, pp. 101-115, 2006.

[40] S. E. Omosigho, "Determination of outdate and shortage quantities in the inventory problem with fixed lifetime," International Journal of Computer Mathematics, vol. 79, no. 11, pp. 1169-1177, 2002.

[41] M. Held and R. M. Karp, "The traveling-salesman problem and minimum spanning trees," Operations Research, vol. 18, no. 6, pp. 1138-1162, 1970.

[42] A. M. Geoffrion and R. McBride, "Lagrangean relaxation applied to capacitated facility location problems," AIIE Transactions, vol. 10, no. 1, pp. 40-47, 1978.

[43] B. Shetty, "Approximate solutions to large scale capacitated facility location problems," Applied Mathematics and Computation, vol. 39, no. 2, pp. 159-175, 1990. 
[44] I. Al-Harkan and M. Hariga, "A simulation optimization solution to the inventory continuous review problem with lot size dependent lead time," Arabian Journal for Science and Engineering, vol. 32, no. 2 B, pp. 327-338, 2007.

[45] S. Park, T. E. Lee, and C. S. Sung, "A three-level supply chain network design model with risk-pooling and lead times," Transportation Research Part E: Logistics and Transportation Review, vol. 46, no. 5, pp. 563-581, 2010.

[46] M. L. Fisher, "Applications oriented guide to Lagrangian relaxation," Interfaces, vol. 15, no. 2, pp. 10-21, 1985.

[47] L. V. Snyder, M. S. Daskin, and C. P. Teo, "The stochastic location model with risk pooling," European Journal of Operational Research, vol. 179, no. 3, pp. 1221-1238, 2007.

[48] H. Y. Mak and Z. J. M. Shen, "A two-echelon inventory-location problem with service considerations," Naval Research Logistics, vol. 56, no. 8, pp. 730-744, 2009.

[49] M. Daskin, Network and Discrete Location: Models, Algorithms and Applications, Palgrave Macmillan, New York, NY, USA, 1997.

[50] R. Haijema, J. van der Wal, and N. M. van Dijk, "Blood platelet production: optimization by dynamic programming and simulation," Computers and Operations Research, vol. 34, no. 3, pp. 760-779, 2007. 


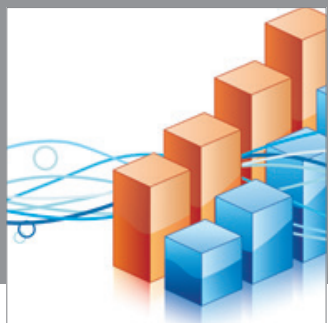

Advances in

Operations Research

mansans

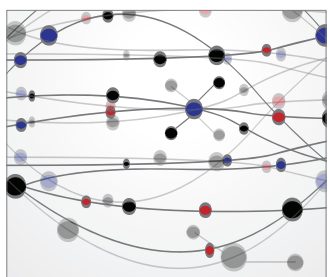

The Scientific World Journal

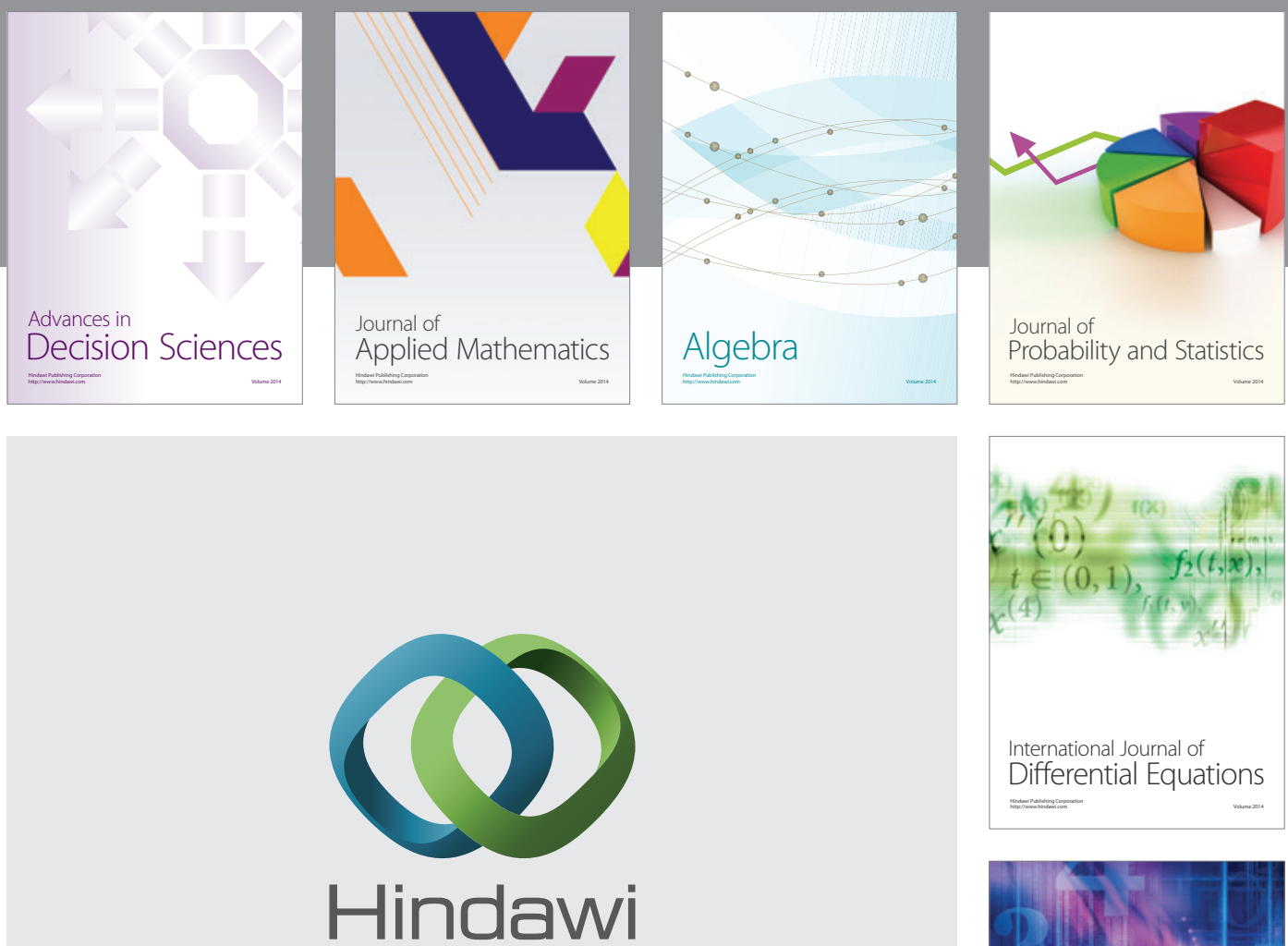

Submit your manuscripts at http://www.hindawi.com
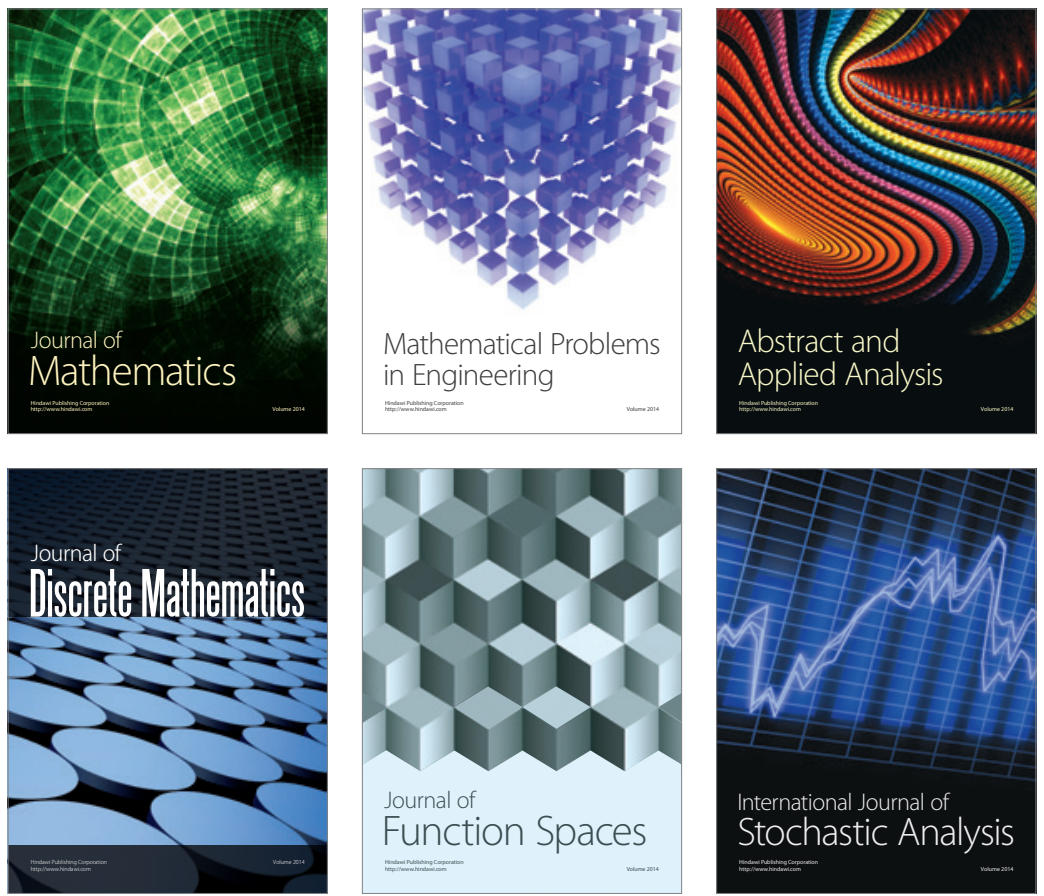

Journal of

Function Spaces

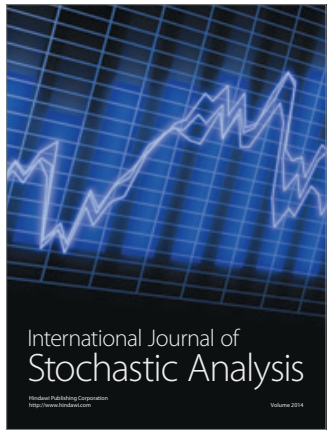

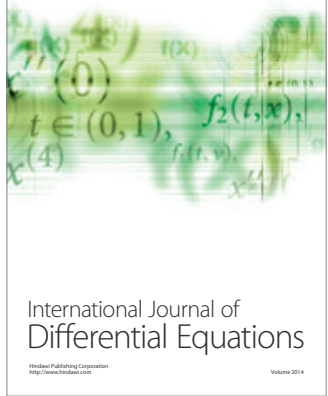
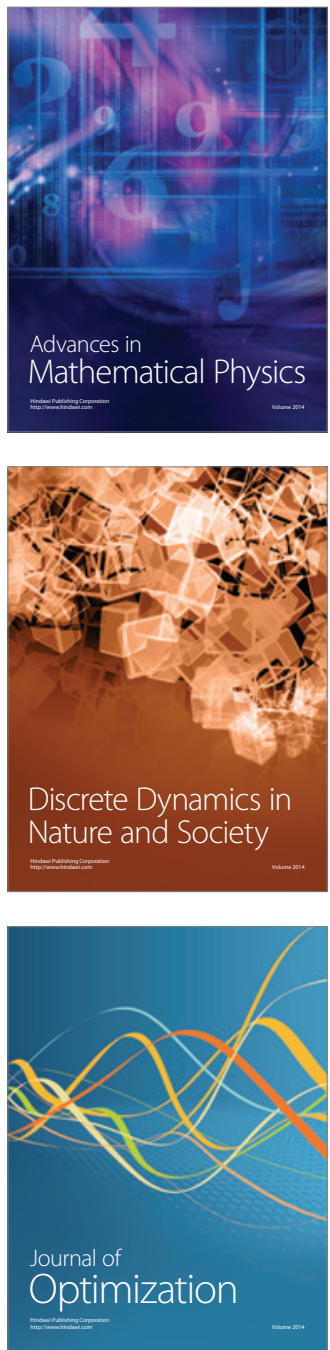